\title{
Mechanical Offset for Torque Ripple Reduction for Magnetless Double-Stator Doubly-Salient Machine
}

\author{
Christopher H. T. Lee ${ }^{1}$, K. T. Chau ${ }^{1}$, Fellow, IEEE, Chunhua Liu ${ }^{1}$, T. W. Ching ${ }^{2}$, and Fuhua $\mathrm{Li}^{1}$ \\ ${ }^{1}$ Department of Electrical and Electronic Engineering, The University of Hong Kong, Hong Kong, China \\ ${ }^{2}$ Department of Electromechanical Engineering, The University of Macau, Macau, China
}

\begin{abstract}
This paper implements the new design structure, so-called the mechanical offset (MO) into the double-stator multitoothed switched reluctance (DS-MSR) machine to form the new MO-DS-MSR machine. The major distinction of the MO structure is to purposely mismatch the outer and inner rotor teeth with a conjugated angle. With the MO structure, the outer and the inner torque components can be compensated with each other, hence minimizing the resultant torque ripple. By employing the finite element analysis, the characteristics and performances of the proposed machines are analyzed and compared.
\end{abstract}

Index Terms - doubly-salient, magnetless, multitoothed, double-stator, mechanical offset, torque ripple reduction.

\section{INTRODUCTION}

$\mathrm{T}$ HERE is an accelerating pace on the development of electric vehicles $(E V s)$ due to the increasing demands on the protection of the environment. Generally speaking, to increase the market penetration of EVs, the EV motor has to offer high efficiency, high power density, high controllability, wide speed range, and maintenance-free operation $[1,2]$. In general cases, the permanent magnet (PM) machines can achieve all these goals and thus these types of machine have been actively developed [3, 4]. However, the prices of the PM materials have risen rapidly [5]; and therefore the advanced magnetless machines, which contain the cost benefits, become more and more attractive.

Compared with the PM machines, the magnetless doublysalient machines suffer from relative poor torque density [6]. In order to resolve this deficiency, the multitoothed switched reluctance (MSR) machine has been proposed and developed [7, 8]. However, the MSR machine utilizes only half of its torque producing zone, and results with the larger torque ripple.

In this paper, by implementing a new design structure, socalled the mechanical offset (MO), into the double-stator (DS) MSR machine, the new MO-DS-MSR machine is proposed. With the proposed design, the outer and inner rotor teeth of the machine are purposely offset with a conjugated angle, such that both stators can contribute powers as the complementary torques with each other. These result with an offset function to produce a smoother resultant torque. By applying the finite element analysis $[9,10]$, the proposed design will be verified and compared.

\section{PROPOSED MACHINES}

Fig. 1(a) shows the topology of the conventional MSR machine, which consists of outer stator of 12 salient poles, each fitted with 2 teeth and results with the equivalent stator teeth of 24. This complies with the rotor of 20 poles. Fig. 1(b) shows the topology of the DS-MSR machine, which obtains the similar structure as the MSR machine does. Meanwhile, the DS-MSR contains the sided-stator sandwiched-rotor structure. The outer and the inner rotor teeth of the DS-MSR

Manuscript received March 6, 2014. Corresponding author: K.T. Chau (e-mail: ktchau@eee.hku.hk).

Digital Object Identifier inserted by IEEE are aligned with each other; hence, its outer and inner armature windings can be connected in series to ease the control algorithm.

Fig. 1(c) shows the topology of the proposed MO-DS-MSR machine, which has the similar topology as the DS-MSR machine does. Meanwhile, its outer and inner rotor teeth are purposely offset with a conjugated angle to each other, as indicated in the circle. Therefore, in order to drive the rotor properly, the outer and the inner windings should be controlled independently.
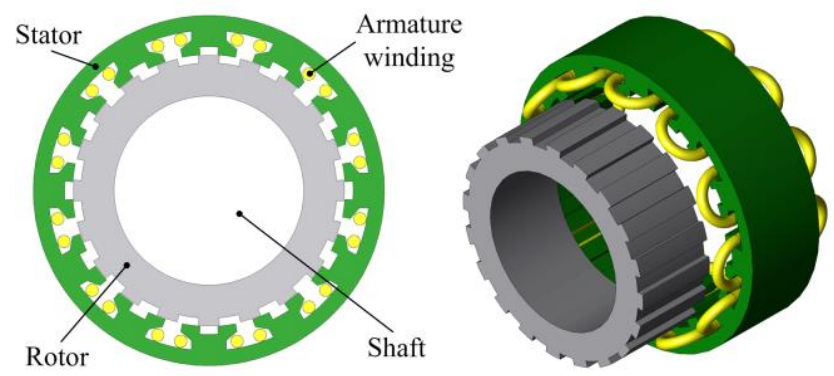

(a)
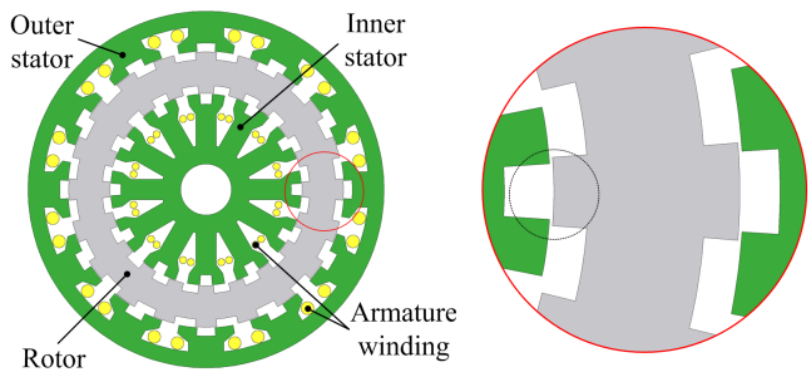

(b)
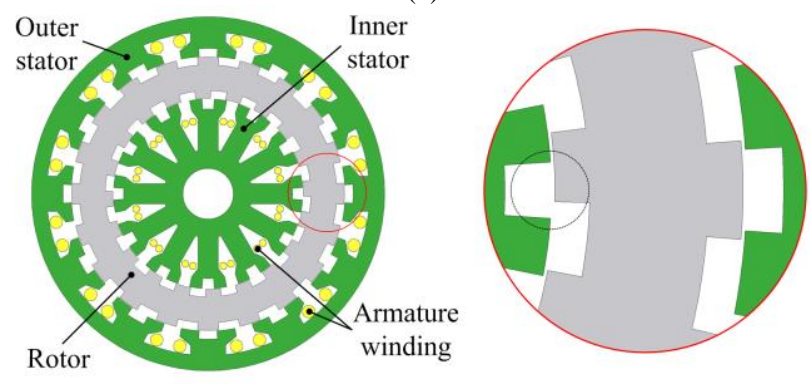

(c)

Fig. 1. Machine configurations: (a) MSR. (b) DS-MSR. (c) MO-DS-MSR. 
The design of the pole-pair arrangement for the machines is based on the following criteria [8]:

$$
\left\{\begin{array}{l}
N_{s p}=2 m j \\
N_{s e}=N_{s p} N_{s t} \\
N_{r}=N_{s e} \pm 2 j
\end{array}\right.
$$

where $N_{s p}$ is the number of stator poles, $N_{s t}$ the stator teeth, $N_{s e}$ the equivalent stator poles, $N_{r}$ the rotor poles, $m$ the armature phases and $j$ is any integer. By selecting $m=3, j=2$ and $N_{s t}=$ 2 , this ends up with $N_{s e}=24, N_{r}=20$, and comes up the proposed machine structures. All machines are designed to avoid the magnetic saturation, and the key design data is shown in Table I.

The key features of the proposed machines are summarized as follows:

- Without installation of any PM materials, the proposed machines enjoy the merit of high cost-effectiveness.

- With the structure of multitoothed per stator pole, the proposed machine can offer the flux-modulation effect to boost up its torque density [7, 8].

- By adopting the DS topology, the inner spacing of the machines can be utilized for further torque enhancement.

- With the MO structure, the outer and inner torque components of the MO-DS-MSR can purposely offset with a conjugated angle to achieve torque ripple minimization.

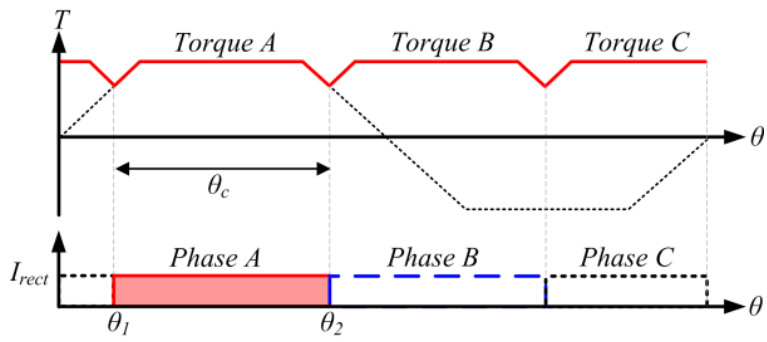

(a)

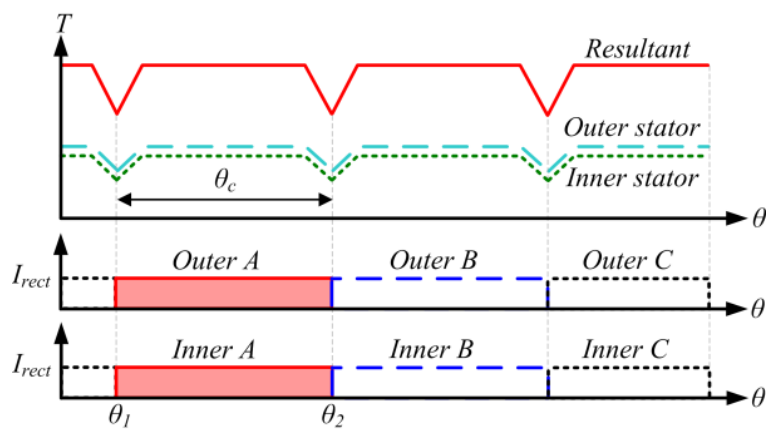

(b)

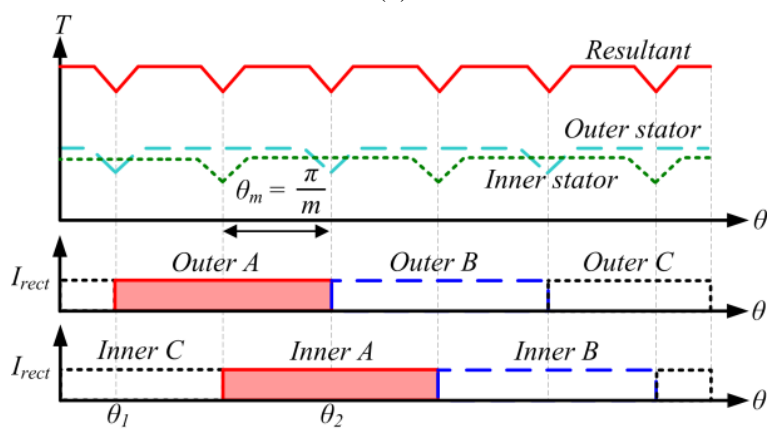

(c)

Fig.2. Operating principles: (a) MSR. (b) DS-MSR. (c) MO-DS-MSR.
TABLE I. KEY DATA OF PROPOSED MACHINES

\begin{tabular}{llll}
\hline \hline Item & MSR & DS-MSR & MO-DS-MSR \\
\hline Outer stator outside diameter & $280.0 \mathrm{~mm}$ & $280.0 \mathrm{~mm}$ & $280.0 \mathrm{~mm}$ \\
Outer stator inside diameter & $217.2 \mathrm{~mm}$ & $217.2 \mathrm{~mm}$ & $217.2 \mathrm{~mm}$ \\
Rotor outside diameter & $216.0 \mathrm{~mm}$ & $216.0 \mathrm{~mm}$ & $216.0 \mathrm{~mm}$ \\
Rotor inside diameter & $151.2 \mathrm{~mm}$ & $151.2 \mathrm{~mm}$ & $151.2 \mathrm{~mm}$ \\
Inner stator outside diameter & N/A & $150.0 \mathrm{~mm}$ & $150.0 \mathrm{~mm}$ \\
Inner stator inside diameter & N/A & $40.0 \mathrm{~mm}$ & $40.0 \mathrm{~mm}$ \\
Air-gap length & $0.6 \mathrm{~mm}$ & $0.6 \mathrm{~mm}$ & $0.6 \mathrm{~mm}$ \\
Stack length & $80.0 \mathrm{~mm}$ & $80.0 \mathrm{~mm}$ & $80.0 \mathrm{~mm}$ \\
No. of stator poles & 12 & 12 & 12 \\
No. of stator teeth & 2 & 2 & 2 \\
No. of equivalent stator poles & 24 & 24 & 24 \\
No. of rotor poles & 20 & 20 & 20 \\
No. of armature phases & 3 & 3 & 3 \\
Mechanical offset & $0^{\circ}$ & $0^{\circ}$ & $60^{\circ}$ \\
No of turns per outer coil & 50 & 50 & 50 \\
No of turns per inner coil & N/A & 60 & 60 \\
\hline \hline
\end{tabular}

\section{MACHINE OPERATIONS}

\section{A. Conventional Conduction Algorithm}

Similar as the conventional SR machines, to drive the MSR machines, a unipolar rectangular current, $I_{\text {rect }}$ is fed to the armature winding during the increasing period of its selfinductance, $L$ such that $\theta_{c}=\theta_{2}-\theta_{1}$, as shown in Fig. 2(a). Under this conduction scheme, the corresponding reluctance torque can be described as $[6,8]$ :

$T=\frac{1}{2} I_{\text {rect }}{ }^{2} \frac{d L}{d \theta}$

It can be seen that the averaged rated torque is governed by the armature current value, which is also regulated by the machine design. Even though the torque ripple value is inversely proportional to the averaged rated torque, the increase of the armature current may also enlarge the torque ripple value and offset the effect of the increased rated torque. Hence, by purely regulating the armature current, it results insignificant improvement towards the torque ripple problem.

Conventionally, for the DS machine, its two stators are operated simultaneously with no phase shift, as shown in Fig. 2(b), and each armature phase can be described as:

$\left\{\begin{array}{lll}i_{k}=I_{\text {rect }} & \theta_{1} \leq \theta \leq \theta_{2} & \text { for } k=1,2 \\ i_{k}=0 & 0 \leq \theta \leq \theta_{1}, \theta_{2} \leq \theta \leq 2 \pi\end{array}\right.$

where $i_{1}$ is the outer armature current and $i_{2}$ the inner armature current. With this conduction scheme, the two stators can transfer the energy to the rotor simultaneously to boost up its torque density. However, the local maxima and local minima of the two torque components are constructively interfered with each other. Hence, with the conventional structure, the DS machine can enjoy only the torque enhancement advantage, but still has no significant improvement regarding to the torque ripple issue.

\section{B. Proposed Mechanical Offset Algorithm}

According to the equation (2), the torque value is governed by the relative position among the stator and the rotor teeth. Hence, the local maxima and the local minima are also governed by the relative stator-rotor position. With the proposed MO structure, the outer and the inner rotor teeth of the MO-DS-MSR machine are purposely mismatched with a conjugated electrical angle of $\theta_{m}=\pi / m$ as shown in Fig. 2(c). The two stators should be operated independently, in which 
one set of them adopts the conventional conduction algorithm as equation (3), and the other set adopts the following:

$$
\begin{cases}i_{1 \text { or } 2}=I_{\text {rect }} & \theta_{1}+\theta_{m} \leq \theta \leq \theta_{2}+\theta_{m} \\ i_{1_{\text {or } ~} 2}=0 & \theta_{m} \leq \theta \leq \theta_{1}+\theta_{m}, \theta_{2}+\theta_{m} \leq \theta \leq 2 \pi+\theta_{m}\end{cases}
$$

With this special mismatched arrangement, the local maxima and the local minima are offset with each other. Hence, the torque ripples from the outer and the inner torque components can be compensated; hence producing the smoother resultant torque. In order to offer the better performance, the magnitudes of the two torque ripples should be adjusted as the similar level with the conjugated patterns. The torque ripple value and pattern can be regulated by the machine design, the armature current magnitude, the winding arrangement, etc. However, the further study of the torque ripple characteristics is out of the scope of this paper.

Since the two torque components are complimented with each other, its averaged value should not be deteriorated. Therefore, with the proposed MO design, the DS machine can still offer the same torque level as those from the conventional one. Since the local maxima and the minima are distributed with the conjugated positions, the resultant torque under the MO design engages with higher ripple frequency. Meanwhile, the armature currents of the MO-DS-MSR are the same as the conventional one. Thus, its copper loss should be similar as its conventional counterpart.

\section{MACHINE PERFORMANCE ANALYSIS}

By performing the finite element analysis, the performances of the proposed machines can be calculated and analyzed. Firstly, the averaged torque and the torque ripple performances of the MSR, the DS-MSR, and the MO-DSMSR machines are as shown in Fig. 3. It can be observed that the average torques of all three machines rising up with the increased armature currents, fulfilling the description of the equation (2). As explained, compared with the MSR, the improved torque density of the DS-MSR has little influence towards the torque ripple values. Meanwhile, by adopting the proposed MO structure, the MO-DS-MSR machine can greatly minimize its torque ripple level compared with its counterpart.

Secondly, the steady torque waveforms of the MSR, the DSMSR and the MO-DS-MSR machines are shown in Fig. 4. With the armature excitation of $10 \mathrm{~A}$, it can be seen that the average steady torques of the MSR, the DS-MSR and the MODS-MSR are $12.9 \mathrm{Nm}, 25.9 \mathrm{Nm}$, and $25.7 \mathrm{Nm}$, respectively. The results confirm that the two torque components of the MO-DS-MSR can integrate perfectly and reach the same torque level as those with the conventional design.

Thirdly, as shown in Fig. 4(b), based on the conventional design, the local maxima and the local minima of the outer and the inner torque ripples are addictively integrated. This offsets the effect from the risen average torque and results with the torque ripple of $52.4 \%$, as comparable to those from the MSR machine of $51.6 \%$, as shown in Fig. 4(a) Hence, the DS-MSR machine gains no benefit according to the torque ripple issue. Meanwhile, as shown in Fig. 4(c), with the proposed MO design, the local maxima and the local minima are purposely mismatched and compensated with each other.
Hence, the MO-DS-MSR machine can enjoy the whole benefit from the increased torque density and result with an improved torque ripple of $27.9 \%$. In addition, it also shows that even with the same operating speed, the MO-DS-MSR machine results an increased torque ripple frequency.

Finally, the copper losses of the machines are shown in Fig. 5. The average copper losses of the MSR, the DS-MSR and the MO-DS-MSR are $111 \mathrm{~W}, 221 \mathrm{~W}$, and $223 \mathrm{~W}$, respectively. These confirm the proposed MO structure has no negative effect towards the copper loss. In addition, all the copper losses are acceptable compared with its power levels.

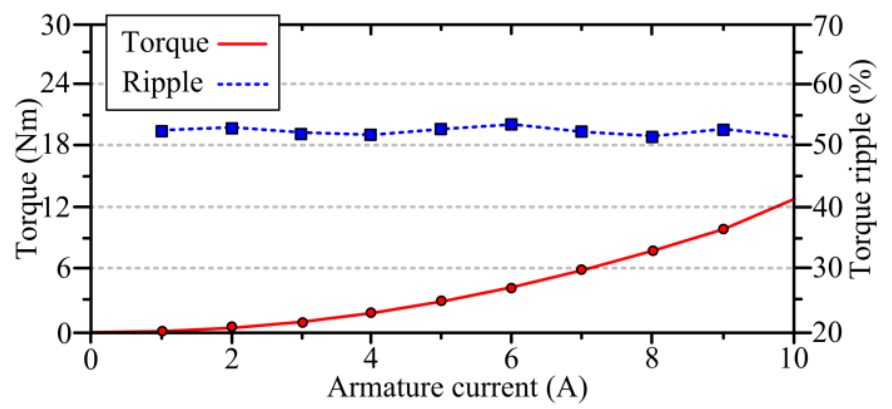

(a)

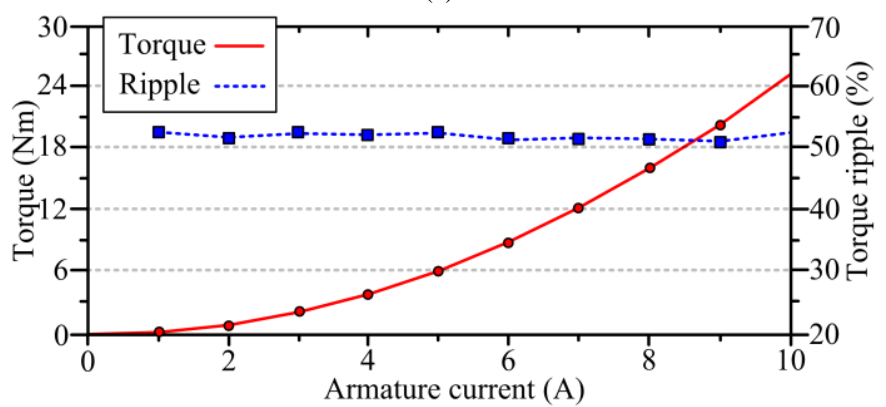

(b)

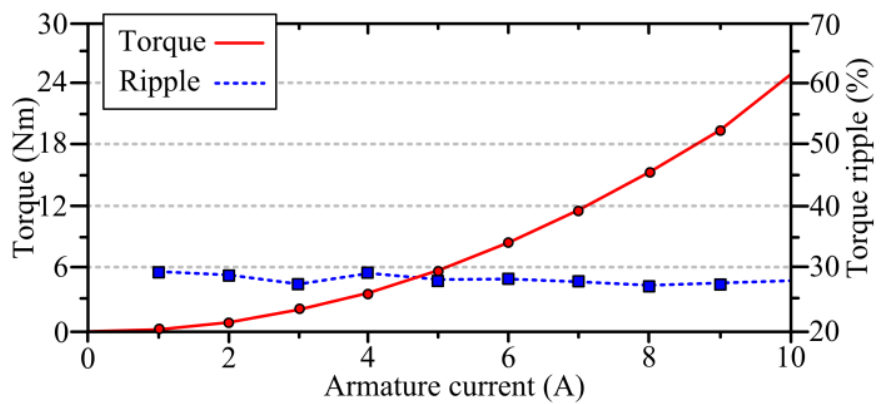

(c)

Fig. 3. Average torque and torque ripple performances: (a) MSR. (b) DSMSR. (c) MO-DS-MSR.

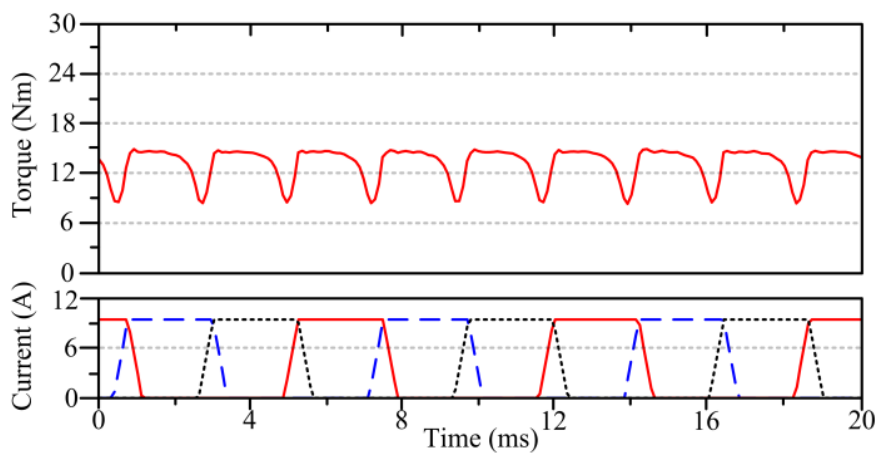

(a) 


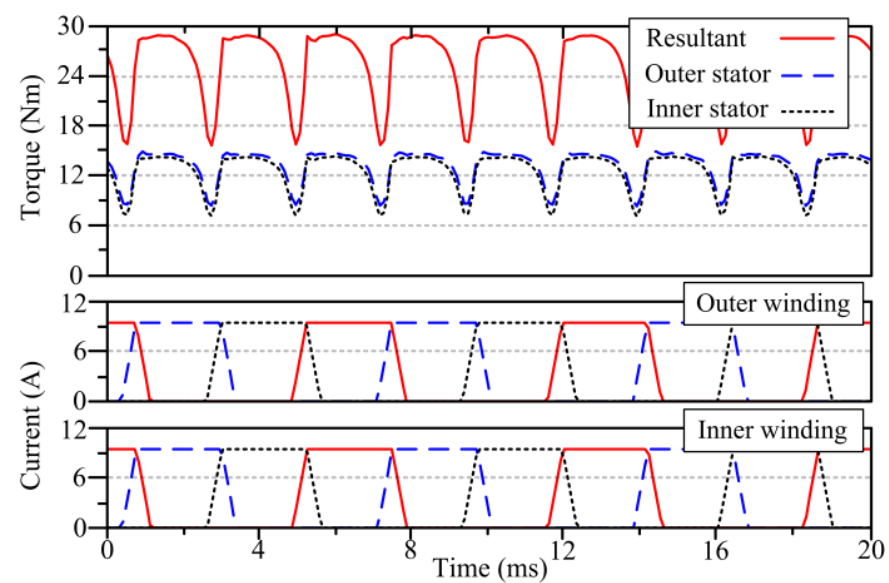

(b)

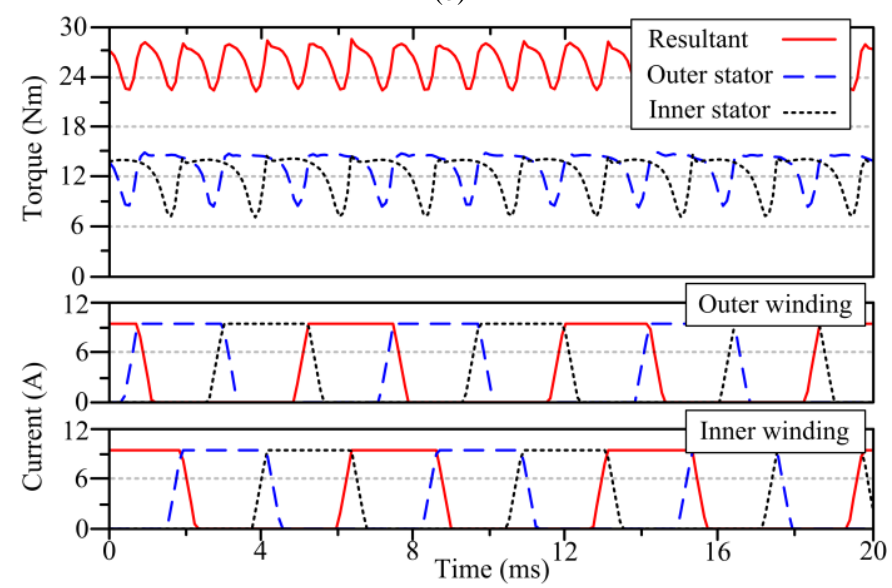

(c)

Fig. 4. Rated torque waveforms: (a) MSR. (b) DS-MSR. (c) MO-DS-MSR.

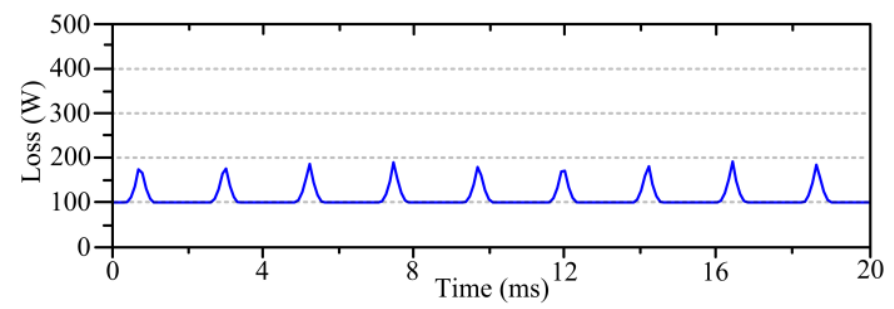

(a)

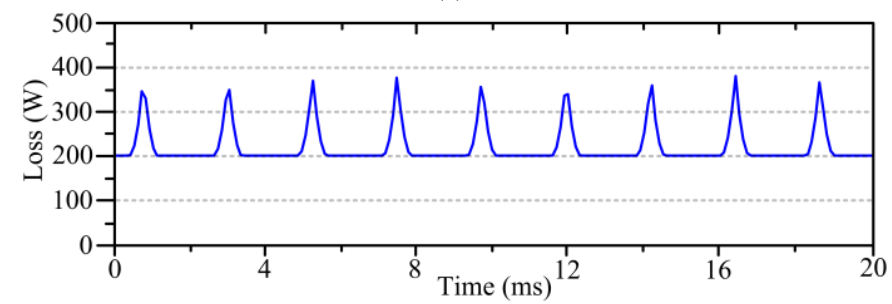

(b)

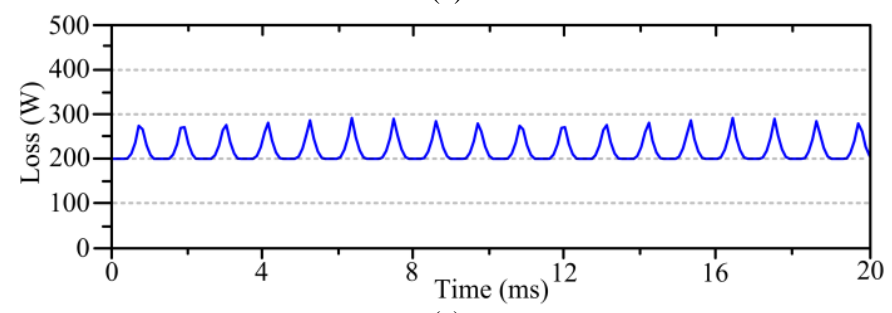

(c)

Fig. 5. Copper loss waveforms: (a) MSR. (b) DS-MSR. (c) MO-DS-MSR.
TABLE II. MACHINE PERFORMANCE COMPARISONS

\begin{tabular}{llll}
\hline \hline Item & MSR & DS-MSR & MO-DS-MSR \\
\hline Power & $620 \mathrm{~W}$ & $1220 \mathrm{~W}$ & $1210 \mathrm{~W}$ \\
Rated speed & $450 \mathrm{rpm}$ & $450 \mathrm{rpm}$ & $450 \mathrm{rpm}$ \\
Outer stator torque & N/A & $13.2 \mathrm{Nm}$ & $13.2 \mathrm{Nm}$ \\
Inner stator torque & N/A & $12.7 \mathrm{Nm}$ & $12.7 \mathrm{Nm}$ \\
Resultant torque & $13.2 \mathrm{Nm}$ & $25.9 \mathrm{Nm}$ & $25.7 \mathrm{Nm}$ \\
Torque ripple & $51.6 \%$ & $52.4 \%$ & $27.9 \%$ \\
Copper loss & $111 \mathrm{~W}$ & $221 \mathrm{~W}$ & $223 \mathrm{~W}$ \\
\hline \hline
\end{tabular}

\section{CONCLUSION}

In this paper, the new torque ripple minimized MO-DSMSR machine is presented. The performances are analyzed by the TS-FEM and the comparison results are summarized in Table II, and concluded as below:

1) Beneficial from the DS topology, the proposed machine can offer higher torque density than its counterpart.

2) With the conventional rotor teeth arrangement, the outer and the inner torque ripples are unfavorably integrated and offset the effect from the increased averaged torque.

3) The proposed MO structure can purposely mismatch and compensate the torque ripples; hence, allowing the DS machine to enjoy all the benefits.

4) The proposed doubly-salient machines obtain no PM materials, thus having better cost-effectiveness than the PM machines do.

\section{ACKNOWLEDGMENT}

This work was supported by a grant (Project No. HKU710612E) from the Hong Kong Research Grants Council, Hong Kong Special Administrative Region, China.

\section{REFERENCES}

[1] Z. Q. Zhu, and D. Howe "Electrical machines and drives for electric, hybrid, and fuel cell vehicles" IEEE Proc., vol. 95, no. 4, pp. 746-765, Apr. 2007.

[2] K. T. Chau., W. Li, and C. H. T. Lee, "Challenges and opportunities of electric machines for renewable energy," Progr. Electromagn. Res. B, vol. 42, pp. 45-74, 2012.

[3] C. Liu, K. T. Chau, J. Z. Jiang, and S. Niu "Comparison of statorpermanent-magnet brushless machines," IEEE Trans. Magn., vol. 44, no. 11, pp. 4405-4408, Nov. 2008.

[4] M. Cheng, W. Hua, J. Zhang, and W. Zhao, "Overview of statorpermanent magnet brushless machines," IEEE Trans. Ind. Electron, vol. 58, no. 11, pp. 5087-5101, Nov. 2011.

[5] M. Chen, K. T. Chau, W. Li, and C. Liu, "Cost-effectiveness comparison of coaxial magnetic gears with different magnet materials," IEEE Trans. Magn., vol. 50, no. 2, pp. 7020304, Feb. 2014.

[6] C. H. T. Lee, K. T. Chau, C. Liu, and Q. Chun, "Design and analysis of a new multitoothed magnetless doubly salient machine," IEEE Trans. Appl. Supercond., vol. 24, no. 3, pp. 5200804, Jun. 2014.

[7] J. Faiz, J. W. Finch and H. M. B. Metwally, "A novel switched reluctance motor with multiple teeth per stator pole and comparison of such motors," Electric Power Syst. Res., vol. 34, no. 3, pp. 197-203, Sept. 1995.

[8] C. H. T. Lee, K. T. Chau, C. Liu, D. Wu, and S. Gao, "Quantitative comparison and analysis of magnetless machine with reluctance topologies," IEEE Trans. Magn., vol. 49, no. 7, pp. 3969-3972, Jul. 2013.

[9] S. J. Salon, Finite Element Analysis of Electrical Machines. Boston, U.S.A.: Kluwer Academic Publishers, 1995.

[10] J. Chen, C. V. Nayar, and L. Xu, "Design and finite-element analysis of an outer-rotor permanent magnet generator for directly coupled wind turbines," IEEE Tran. Magn., vol. 36, no. 5, pp. 3802-3809, Sept. 2000. 